\title{
Cocaine-associated atypical haemolytic uraemic syndrome in a genetically susceptible individual
}

Running title: Atypical haemolytic uraemic syndrome

Authors: Elisa Bongetti ${ }^{1}$ David Kavanagh ${ }^{2,3}$, Kylie Martin ${ }^{1}$, Anna Bendall ${ }^{1}$, Prue Hill $^{1}$, Adam Steinberg ${ }^{1}$ Yogeshwar Rajaram ${ }^{1}$, Francesco Ierino ${ }^{1}$

1. Department of Nephrology, St. Vincent’s Hospital, Melbourne, Australia.

2. Institute of Cellular Medicine, Newcastle University, Newcastle upon Tyne, United Kingdom.

3. The National Renal Complement Therapeutics Centre (NRCTC), Newcastle upon Tyne Hospitals NHS Foundation Trust, Newcastle upon Tyne, United Kingdom.

Correspondence: Dr. Elisa Bongetti ${ }^{1}$

elisabongetti@gmail.com

0431844967

Conflict of interest: Newcastle University has received honoraria for consultancy work by

D.K. from Alexion Pharmaceuticals.

This is the author manuscript accepted for publication and has undergone full peer review but has not been through the copyediting, typesetting, pagination and proofreading process, which may lead to differences between this version and the Version of Record. Please cite this article as doi: $10.1111 /$ nep.13690

This article is protected by copyright. All rights reserved. 
1. Current address for correspondence: Renal Unit, Launceston General Hospital, Charles Street, Launceston, Tasmania, 7250

\begin{abstract}
Atypical haemolytic uraemic syndrome (aHUS) is a severe, life-threatening condition that requires early recognition and urgent treatment. In aHUS rare genetic variants in $C F H, C F I, C D 46, C 3$ and $C F B$ predispose to complement over activation. This case describes a case of aHUS in which there was a strong temporal association between disease onset and the use of smoked cocaine. The patient was found to have a rare genetic variant in the CFI gene which may have been unmasked by first-time exposure to cocaine. The patient stabilised and improved with early administration of eculizumab, supporting the notion of an underlying immunological pathogenesis and the importance of early intervention.
\end{abstract}

Key Words: thrombotic microangiopathies, Atypical Hemolytic Uremic Syndrome, Cocaine, Complement Inactivating Agents, Complement Activation

Case History: A forty-seven-year-old man presented to the emergency department with a twoweek history of worsening headache, blurred vision, subjective fevers and orthopnoea. The initial onset of these symptoms occurred within forty-eight hours of smoking cocaine. It was the patient's first exposure to this drug. The patient denied gastrointestinal or infective symptoms. His past medical history was significant for previous heroin intravenous drug use; however, he had abstained for five years. He had chronic Hepatitis B which he acquired as a young child in South-East Asia where he was born and never had treatment with interferon. The patient had no known history of autoimmune disease and had no significant past surgical 
history. He was not administering any regular medication, herb, supplement or complementary medicine, and had no known drug allergies.

On examination, the patient was alert, oriented and afebrile. The blood pressure was 205/150 mmHg. His pulse rate was regular at 100 beats/min and his oxygen saturation on room air was 94\%. The jugular venous pressure was elevated and there were bibasal crackles on chest auscultation. The patient was oligouric.

Results: Initial investigations demonstrated thrombocytopenia (platelets 23 x10 $/ \mathrm{L}$ [150-400 x10 $/ \mathrm{L}]$ ) and a non-immune haemolytic anaemia: (haemoglobin $95 \mathrm{~g} / \mathrm{L}$ [130-180 g/L]) with fragments on the blood film, low haptoglobin $(<0.1 \mathrm{~g} / \mathrm{L}$ [0.3-2.0 g/L]), an elevated LDH (1181 $\mathrm{U} / \mathrm{L}[<250] \mathrm{U} / \mathrm{L}$ ) and a negative direct Coombs test. Acute kidney injury was present and was severe (creatinine $1155 \mu \mathrm{mol} / \mathrm{L}$ [64-104 $\mu \mathrm{mol} / \mathrm{L}]$, urea $39.4 \mathrm{mmol} / \mathrm{L}$ [2.1-7.1 $\mathrm{mmol} / \mathrm{L}]$ ). Complement studies showed low C3 levels (0.69 g/L [0.90-1.80 g/L]) but normal C4 levels. Coagulation screen demonstrated a normal INR (1.1 [0.8-1.2]), normal APTT (32 [26-37]) and fibrinogen was not suppressed (5.3 g/L [2.2-4.0]). Serial cardiac troponins were elevated and peaked at $2013 \mathrm{H}$ ng/L [<26]. An ECG demonstrated widespread t-wave inversion. A transthoracic echocardiogram at the time showed an ejection fraction $50 \%$ with mild to moderate concentric increase in the left ventricular wall thickness. He had moderate pulmonary hypertension (right ventricular systolic pressure $64 \mathrm{mmHg}$ ).

He also had a non-contrast computerised tomography (CT) of the chest which demonstrated changes consistent with pulmonary oedema and a small pericardial effusion. CT

This article is protected by copyright. All rights reserved. 
of the abdomen and pelvis, including renal tract morphology, was unremarkable. The left kidney measured $10 \mathrm{~cm}$, and the right kidney $9 \mathrm{~cm}$.

Further investigations revealed negative serologies for HIV and Hepatitis C but was positive for chronic Hepatitis B infection. Interferon gamma release assay was negative for latent tuberculosis and a comprehensive autoimmune and vasculitic screen (dsDNA, ENA, ANA, ANCA, anti-GBM, anti-PLA2R) was negative. Stools were negative for Shiga toxin PCR. HbA1c was normal (3.6\%) and myeloma screen was negative. An urgent ADAMTS 13 result within 24 hours demonstrated a normal level (44.1\% [40-130]). Genetic testing for complement mutations and autoantibodies to complement regulators was also performed.

Management: Within four hours of presentation to the emergency department the patient was transferred to the intensive care unit where he received immediate renal haemofiltration and plasma exchange with fresh frozen plasma. His blood pressure was gradually controlled with a glycerol trinitrate infusion to a systolic blood pressure of $160 \mathrm{mmHg}$. The following day, with normal ADAMTS 13 result, plasma exchange was ceased and treatment with eculizumab, a humanised anti-complement protein C5 monoclonal antibody was commenced. Prior to eculizumab being given, the patient was immunised with pneumococcal and meningococcal vaccinations and started on a 2-week course of oral amoxicillin for meningococcal prophylaxis. He initially received empirical treatment for hepatitis B with entecavir, however this was ceased as the risk of re-activation was deemed low.

This article is protected by copyright. All rights reserved. 
Progress: Following the initial treatment with eculizumab and concurrent blood pressure control there was a rapid improvement in the patient's thrombocytopenia. However, his renal function remained persistently impaired and he required ongoing maintenance renal replacement therapy with haemodialysis three times per week. The patient was counselled on the importance of future abstinence from cocaine. Bilateral hypertensive retinopathy was diagnosed on eye examination and the patient was continued on antihypertensive medications.

The patient was discharged home after two weeks and continued haemodialysis three times per week with regular infusions of eculizumab as per the manufacturer's product information. A renal biopsy (for prognostic indication) after one month showed vascular changes consistent with chronic TMA with moderate glomerular ischaemic change and moderate chronic tubulointerstitial damage (Figure 1). There was no evidence of ongoing acute thrombotic microangiopathy.

Six months following his initial presentation, the patient was weaned off haemodialysis. Soon after, a considered clinical decision was made to cease treatment with eculizumab, as the patient's blood pressure was adequately controlled, and he had abstained from further cocaine use. His renal function remained impaired but stable (creatinine $300 \mu \mathrm{mol} / \mathrm{L}$ [64-104 $\mu \mathrm{mol} / \mathrm{L}]$ ).

Long-term management was completed by genetic testing and seeking expert advice. The patient was heterozygous for a rare variant (c.1268C>A) in the complement factor I (CFI) gene. The mutation, Ala423Glu, is in direct opposition to C3b (see Figure 2). In silico analysis using PolyPhen2, SIFT and MutationTaster could not exclude pathogenicity. Convalescent 
complement analysis demonstrated normal factor I levels $21 \mathrm{mg} / \mathrm{mL}$ (reference range: 21-40 $\mathrm{mg} / \mathrm{mL}$ ) but continued low C3 (0.6g/L [0.68 to 1.80$])$.

Discussion: aHUS is a rare, progressive, life-threatening form of thrombotic microangiopathy (TMA) characterised by thrombocytopaenia, renal impairment and non-autoimmune haemolytic anaemia $(1,2)$. aHUS is differentiated from thrombotic thrombocytopaenic purpura and Shiga- toxin-producing Escherichia coli haemolytic uraemic syndrome with $>5 \%$ ADAMTS13 activity and the absence of Shiga toxin (3). However, there is no gold standard test for aHUS diagnosis in the acute setting and our case highlights this challenge. There was a clear temporal association between the use of cocaine and the onset of the patient's symptoms. A rare complement factor I (CFI) genetic variant was uncovered with uncertain functional significance, posing unclear benefits of extended treatment of the patient with eculizumab.

Cocaine is a commonly abused stimulant drug that comes in the form of either a hydrochloride salt that is inhaled, or as a free base crystal that is smoked (4). Cocaine is known to cause direct endothelial dysfunction and promote coagulation by activating platelets, increasing levels of von Willebrand factor and fibrinogen (5-7). Cocaine has been reported to cause TMA, either with or without concomitant hypertension $(5,8,9)$. The patient described had evidence of longstanding hypertension on echocardiogram, and CKD (elevated PTH, left ventricular hypertrophy and moderate chronic tubulointerstitial damage on biopsy). Thus, the cocaine may have precipitated malignant phase hypertension in an individual with pre-existing hypertension or may have directly activated the complement system resulting in complement mediated aHUS. 
In complement-mediated aHUS, rare genetic variants in $C F H, C F I, C D 46, C 3, C F B$ and autoantibodies to factor $\mathrm{H}$ are reported to cause complement over activation. These genetic variants predispose, and disease is often unmasked by an environmental trigger (e.g., pregnancy or infection) (10). Our patient was found to be heterozygous for the c.1268C $>\mathrm{A}$ p.(Ala423Glu) variant in the CFI gene. Using bioinformatic analysis this variant is classified as possibly damaging and analysis of the crystal structure demonstrates that the rare genetic variant is adjacent to is substrate, C3b. Thus, it is possible this rare genetic variant impairs CFI function. Moreover, in the reported literature the factor I levels were normal, as in our patient, suggesting that this rare variant does not lead to quantitative deficiency but instead may produce a qualitative defect.

Mutation screening in aHUS is challenging as many of the disease-associated mutations are private, and a significant proportion of variants consist of missense mutations of unknown significance (11). There is enrichment of rare genetic variants in CFI in aHUS although around $2.3 \%$ of control populations will carry these variants $(12,13)$. The mutations described in aHUS are all heterozygous to date, and account for around $10 \%$ of cases of complement-mediated aHUS (14-16). The penetrance of aHUS in individuals with CFI variants is low with few pedigrees demonstrating inheritance. Indeed, functionally significant CFI mutations appear to be a much stronger risk for development of another complement mediated disease; age-related macular degeneration (17). Because of this it is likely that a very potent trigger of aHUS (cocaine and/or hypertension) was required for disease to manifest.

Conclusion: Although genetic analysis can uncover definitively pathogenic mutations which predispose to aHUS the interpretation of variants of unknown significance remains a major 
future challenge. Furthermore, the absence of a gold standard diagnostic test for acute aHUS poses challenges for commencing early urgent intervention with eculizumab which is known to reduce mortality, increases likelihood of renal recovery, and improves quality of life.

\section{References}

1. Kaartinen K, Martola L, Meri S. Atypical hemolytic-uremic syndrome. Duodecim. 2017;133(6):539-47.

2. Brocklebank V, Kavanagh D. Complement C5-inhibiting therapy for the thrombotic microangiopathies: accumulating evidence, but not a panacea. Clinical kidney journal. 2017;10(5):600-24.

3. Hossain MA, Cheema A, Kalathil S, Bajwa R, Costanzo EJ, Cosentino J, et al. Atypical hemolytic uremic syndrome: Laboratory characteristics, complement-amplifying conditions, renal biopsy, and genetic mutations. Saudi journal of kidney diseases and transplantation : an official publication of the Saudi Center for Organ Transplantation, Saudi Arabia. 2018;29(2):276-83.

This article is protected by copyright. All rights reserved. 
4. Takitane J, Leyton V, Andreuccetti G, Gjerde H, Vindenes V, Berg T. Determination of cocaine, metabolites and a crack cocaine biomarker in whole blood by liquid-liquid extraction and UHPLC-MS/MS. Forensic science international. 2018;289:165-74.

5. Dejman A, Alavi SN, Thomas DB, Stefanovic A, Asif A, Nayer A. The potential role of complements in cocaine-induced thrombotic microangiopathy. Clin Kidney J. 2018;11(1):26-8.

6. Pellegrino T, Bayer BM. In vivo effects of cocaine on immune cell function. Journal of neuroimmunology. 1998;83(1-2):139-47.

7. Fiala M, Gan XH, Zhang L, House SD, Newton T, Graves MC, et al. Cocaine enhances monocyte migration across the blood-brain barrier. Cocaine's connection to AIDS dementia and vasculitis? Advances in experimental medicine and biology. 1998;437:199-205.

8. Lamia R, El Ati Z, Ben Fatma L, Zouaghi K, Smaoui W, Rania K, et al. Malignant hypertension-associated thrombotic microangiopathy following cocaine use. Saudi journal of kidney diseases and transplantation : an official publication of the Saudi Center for Organ Transplantation, Saudi Arabia. 2016;27(1):153-6.

9. Duran CE, Blasco M, Maduell F, Campistol JM. Rescue therapy with eculizumab in a transplant recipient with atypical haemolytic-uraemic syndrome. Clin Kidney J. 2012;5(1):2830.

10. Caprioli J, Noris M, Brioschi S, Pianetti G, Castelletti F, Bettinaglio P, et al. Genetics of HUS: the impact of MCP, CFH, and IF mutations on clinical presentation, response to treatment, and outcome. Blood. 2006;108(4):1267-79.

This article is protected by copyright. All rights reserved. 
11. Kavanagh D, Anderson HE. Interpretation of genetic variants of uncertain significance in atypical hemolytic uremic syndrome. Kidney international. 2012;81(1):11-3.

12. Osborne AJ, Breno M, Borsa NG, Bu F, Fremeaux-Bacchi V, Gale DP, et al. Statistical Validation of Rare Complement Variants Provides Insights into the Molecular Basis of Atypical Hemolytic Uremic Syndrome and C3 Glomerulopathy. Journal of immunology (Baltimore, Md : 1950). 2018;200(7):2464-78.

13. Seddon JM, Yu Y, Miller EC, Reynolds R, Tan PL, Gowrisankar S, et al. Rare variants in CFI, C3 and C9 are associated with high risk of advanced age-related macular degeneration. Nature genetics. 2013;45(11):1366-70.

14. Kavanagh D, Richards A, Noris M, Hauhart R, Liszewski MK, Karpman D, et al. Characterization of mutations in complement factor I (CFI) associated with hemolytic uremic syndrome. Molecular Immunology. 2008;45(1):95-105.

15. Wong EKS, Kavanagh D. Diseases of complement dysregulation-an overview. Semin Immunopathol. 2018;40(1):49-64.

16. Kavanagh D, Kemp EJ, Mayland E, Winney RJ, Duffield JS, Warwick G, et al. Mutations in complement factor I predispose to development of atypical hemolytic uremic syndrome. Journal of the American Society of Nephrology : JASN. 2005;16(7):2150-5.

17. Kavanagh D, Yu Y, Schramm EC, Triebwasser M, Wagner EK, Raychaudhuri S, et al. Rare genetic variants in the CFI gene are associated with advanced age-related macular degeneration and commonly result in reduced serum factor I levels. Human molecular genetics. 2015;24(13):3861-70.

This article is protected by copyright. All rights reserved. 


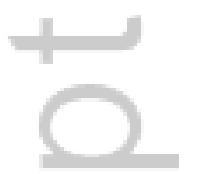

This article is protected by copyright. All rights reserved. 


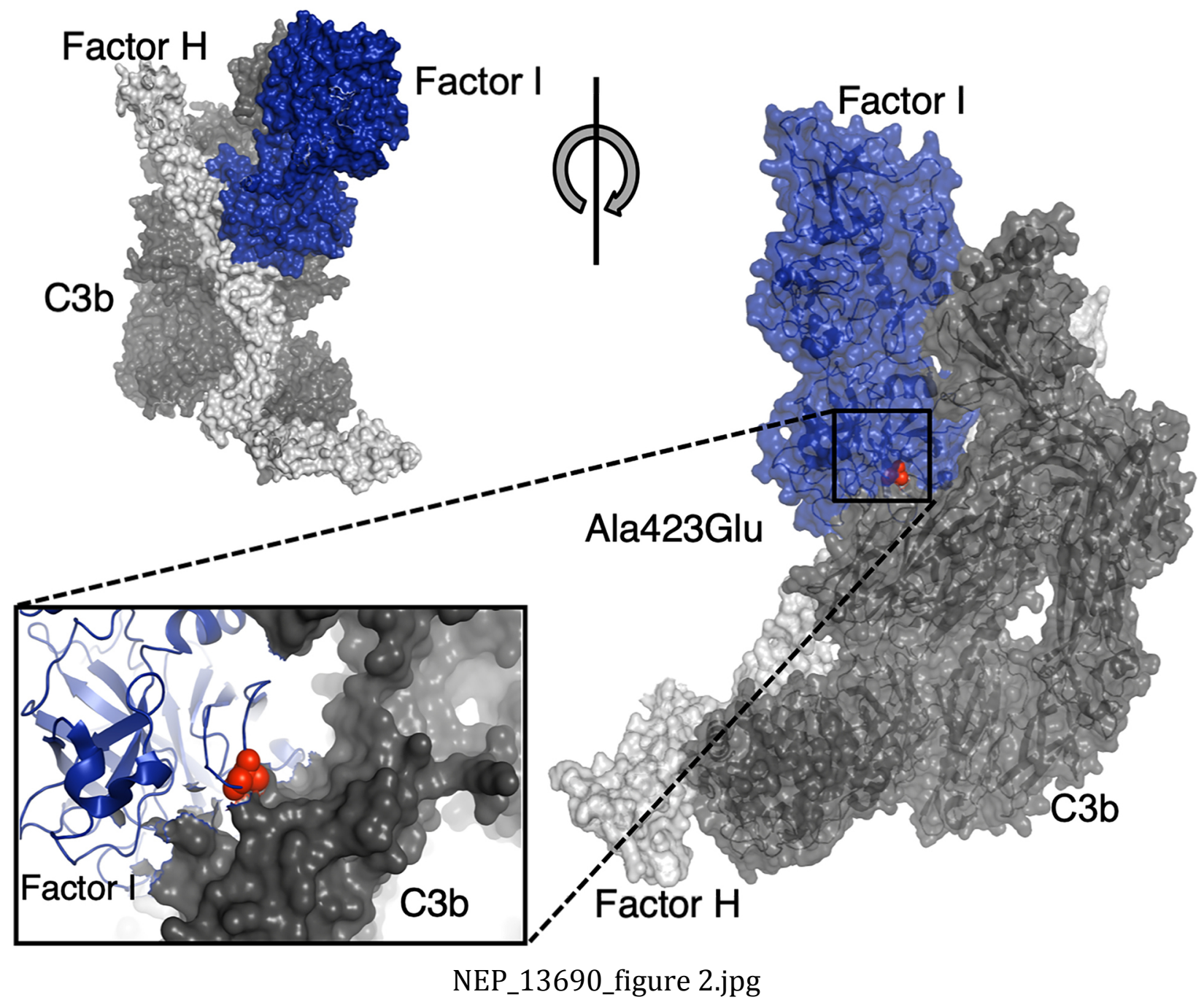

This article is protected by copyright. All rights reserved. 


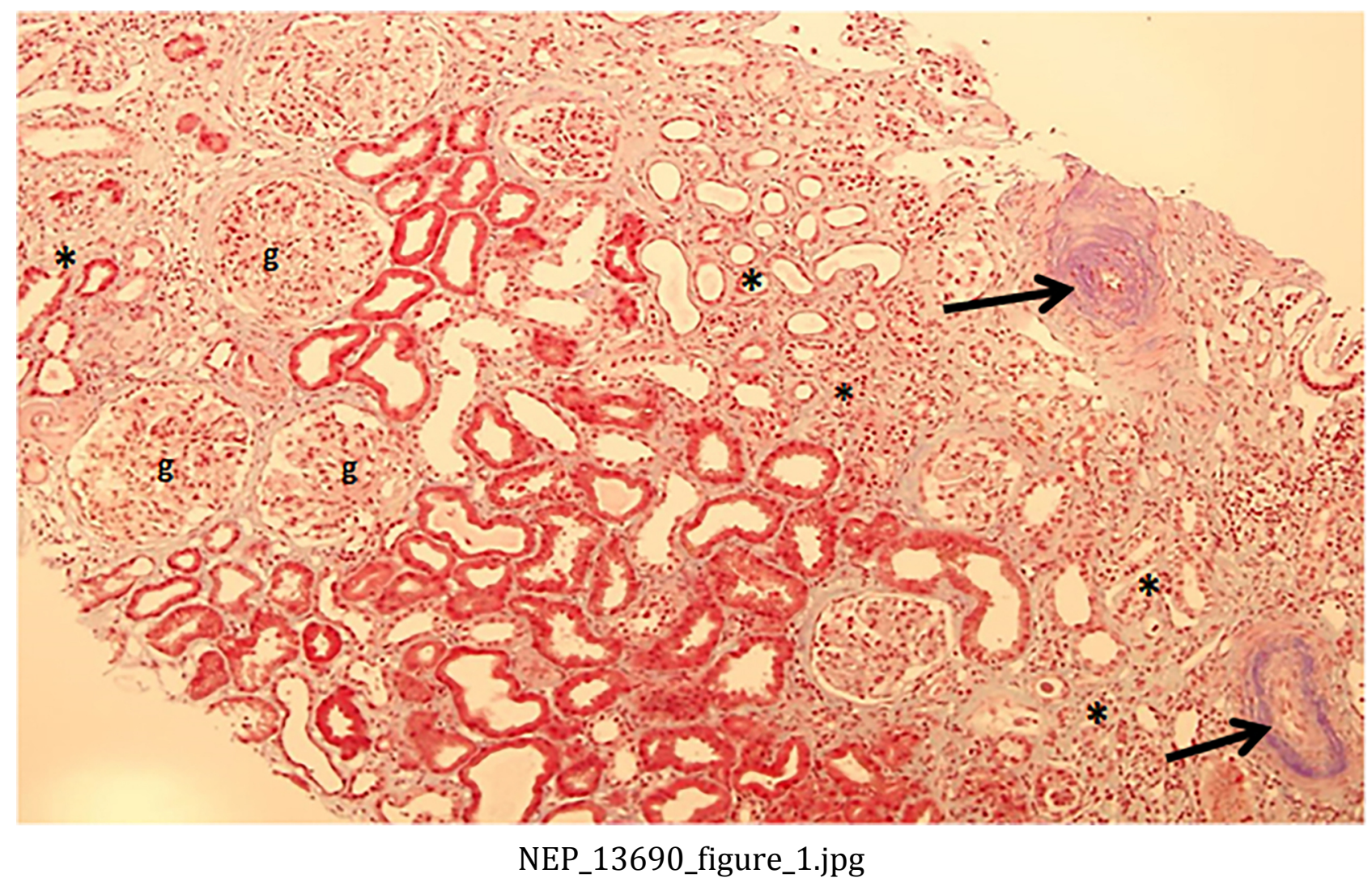

This article is protected by copyright. All rights reserved. 
Cocaine-associated atypical haemolytic uraemic syndrome in a genetically susceptible individual

Running title: Atypical haemolytic uraemic syndrome

Authors: Elisa Bongetti ${ }^{1}$, David Kavanagh ${ }^{2,3}$, Kylie Martin ${ }^{1}$, Anna Bendall ${ }^{1}$, Prue Hill $^{1}$, Adam Steinberg ${ }^{1}$ Yogeshwar Rajaram ${ }^{1}$, Francesco Ierino ${ }^{1}$

1. Department of Nephrology, St. Vincent’s Hospital, Melbourne, Australia.

2. Institute of Cellular Medicine, Newcastle University, Newcastle upon Tyne, United Kingdom.

3. The National Renal Complement Therapeutics Centre (NRCTC), Newcastle upon Tyne Hospitals NHS Foundation Trust, Newcastle upon Tyne, United Kingdom.

Correspondence: Dr. Elisa Bongetti ${ }^{1}$

elisabongetti@gmail.com

0431844967

Conflict of interest: Newcastle University has received honoraria for consultancy work by D.K. from Alexion Pharmaceuticals.

1. Current address for correspondence: Renal Unit, Launceston General Hospital, Charles Street, Launceston, Tasmania, 7250

This article is protected by copyright. All rights reserved. 


\section{University Library}

\section{- M M N E R VA A gateway to Melbourne's research publications}

Minerva Access is the Institutional Repository of The University of Melbourne

Author/s:

Bongetti, E;Kavanagh, D;Martin, K;Bendall, A;Hill, P;Steinberg, A;Rajaram, Y;lerino, F

Title:

Cocaine-associated atypical haemolytic uraemic syndrome in a genetically susceptible individual

Date:

2020-01-17

Citation:

Bongetti, E., Kavanagh, D., Martin, K., Bendall, A., Hill, P., Steinberg, A., Rajaram, Y. \& Ierino, F. (2020). Cocaine-associated atypical haemolytic uraemic syndrome in a genetically susceptible individual. NEPHROLOGY, 25 (7), pp.518-521. https://doi.org/10.1111/ nep. 13690 .

Persistent Link:

http://hdl.handle.net/11343/275275 